\title{
miR-378a-5p regulates CAMKK2/AMPK pathway to contribute to cerebral ischemia/reperfusion injury-induced neuronal apoptosis
}

\author{
Yun Zhang ${ }^{1,2}$, Peilan Zhang ${ }^{1}$, Chunying Deng ${ }^{1,3}$ \\ ${ }^{1}$ Clinical College of Neurology, Neurosurgery and Neurorehabilitation, Tianjin Medical University, \\ 300350, China \\ ${ }^{2}$ Department of Geriatrics, Tangshan Gongren Hospital, Tangshan City, Hebei Province, 063000, China \\ ${ }^{3}$ Department of Neurology, North China University of Science and Technology Affiliated Hospital, \\ Tangshan City, Hebei Province, 063000, China
}

\begin{abstract}
Introduction. The pathological mechanism of cerebral ischemia/reperfusion (CIR) injury is complicated and unclear. Apart from the involvement of many low-molecular factors it was found that several miRNAs were dysregulated during and after CIR injury in cell models. This study aimed to explore the effects of miR-378a$5 \mathrm{p}$ on in vitro model of (CIR) injury-induced neuronal apoptosis and provide a new mechanism of CIR injury. Material and methods. Primary hippocampal neurons were isolated from newborn Sprague-Dawley rats. Oxygen-glucose deprivation/reoxygenation (OGDR) for $24 \mathrm{~h}$ and $48 \mathrm{~h}$ was used as an in vitro model of CIR. Cell viability was measured using MTT assay and apoptosis was determined by flow cytometry. Quantitative real time PCR (qRT-PCR) assay and Western blotting were used to examine mRNA and protein expressions, respectively. TargetScan was used to predict the direct target of miR-378a-5p and luciferase assay was used to validate that calmodulin-dependent protein kinase kinase-2 (CAMKK2) was the direct target of miR-378a-5p.

Results. miR-378a-5p expression was significantly increased after OGDR at $24 \mathrm{~h}$ and $48 \mathrm{~h}$. After OGDR, cell viability was reduced, which was reversed by miR-378a-5p and enhanced by shCAMKK2 plasmid. Cell apoptosis was increased after OGDR, which was prevented by miR-378a-5p and enhanced by shCAMKK2 plasmid. Results of TargetScan and luciferase assay demonstrated that miR-378a-5p could directly bind to 3'-untranslated region (3'-UTR) of CAMKK2. Both mRNA and protein expression of CAMKK2 were downregulated by miR-378a-5p mimics and upregulated by miR-378a-5p inhibitors. Phosphorylation of adenosine monophosphate-activated protein kinase (AMPK) was positively associated with expression of CAMKK2.

Conclusions. Data of this study indicated that miR-378a-5p was significantly overexpressed after OGDR. miR$378 \mathrm{a}-5 \mathrm{p}$ could bind to 3'-UTR of CAMKK2 to inhibit cell proliferation through regulation of CAMKK2/AMPK pathway providing a new mechanism and biomarker for the diagnosis and potential treatment of CIR injury. (Folia Histochemica et Cytobiologica 2021, Vol. 59, No. 1, 57-65)
\end{abstract}

Key words: miR-378a-5p; primary hippocampal neurons; rat; oxygen-glucose deprivation/reoxygenation; CAMKK2/AMPK pathway; apoptosis

Correspondence address: Peilan Zhang

Department of Neurology, Tianjin Huanhu Hospital,

No. 6 Jizhao Road, Jinnan District, Tianjin, China

phone: $86+022-59065241$

e-mail: zhangpeilan666@163.com

\section{Introduction}

The brain is an organ highly sensitive to hypoxia [1]. Cerebral ischemia/reperfusion (CIR) injury occurs when blood supply to the brain is suspended and

This article is available in open access under Creative Common Attribution-Non-Commercial-No Derivatives 4.0 International (CC BY-NC-ND 4.0) license, allowing to download articles and share them with others as long as they credit the authors and the publisher, but without permission to change them in any way or use them commercially. 
subsequently restored [2]. Cerebral IR injury can induce brain dysfunction, which contributes to high mortality and disability [3]. The pathological mechanism of CIR injury is complicated and unclear. Many biological processes are involved in it, including the generation of reactive oxygen species (ROS), reduction of adenosine triphosphate (ATP) and antioxidative factors, including activation of adenosine monophosphate-activated protein kinase (AMPK) signaling pathway $[4,5]$.

The major function of AMPK is to monitor the changes of cellular energy and control the ATP level, thus AMPK activity is regulated by the ratio of AMP/ADP to ATP [6]. Phosphorylation of Thr172 is required for the activation of AMPK, which is directly mediated by serine/threonine kinase LKB1, a tumor suppressor protein [7]. In addition, phosphorylation of AMPK on Thr172 can also be mediated via calmodulin-dependent protein kinase kinase-2 (CAMKK2) in response to calcium flux [8]. Activation of AMPK can promote glucose uptake via activation of Akt-mTOR pathway, inhibit protein and lipid synthesis, and suppress cell growth [9]. AMPK is also involved in CIR injury through multiple signaling pathways, including NF- $\kappa \mathrm{B}$ signaling pathway and NLRP3 inflammasome activation pathway [10,11]. As a class of small non-coding RNAs (28-25 nucleotides), microRNAs (miRNAs) can directly target the 3'-untranslated region (3'-UTR) of mRNAs, thereby downregulating protein expression [12]. Several miRNAs were dysregulated during and after CIR injury in cell models [13]. In vascular smooth muscle cells, miR-378a-5p has been reported to promote cell proliferation and migration through targeting cyclin-dependent kinase 1 (CDK1), a protein that promotes cell cycle entering $M$ phase from G2 phase [14]. Overexpression of miR-378a-5p can directly target 3'-UTR of E2F transcription factor 3 (E2F3), leading to lens epithelial cell apoptosis in cataract [15]. However, no studies have reported the effects of miR-378a-5p on CIR injury. This study aims to investigate the effects of miR-378a-5p on in vitro model of CIR-induced neuronal cell apoptosis and provide a new mechanism of diagnosis and treatment for CIR injury.

\section{Material and methods}

Cell culture, treatment and transfection. Human embryonic kidney 293 (HEK293) cells were obtained from Beijing Smart Treasure Biological Technology Co., Ltd. (Genobio, China) and cultured in DMEM (Procell, Wuhan, China) supplemented with fetal bovine serum (FBS, 10\%, Thermo Fisher, Carlsbad, CA, USA) and $1 \%$, penicillin-streptomycin solution (Solarbio, Beijing, China) with $5 \% \mathrm{CO}_{2}$ at $37^{\circ} \mathrm{C}$.
Primary hippocampal neurons were isolated from newborn Sprague-Dawley rats [16]. Briefly, the hippocampal tissues were cut into small pieces and digested with trypsin-EDTA $(0.25 \%$, STEMCELL, Seattle, WA, USA). The isolated primary hippocampal neurons were seeded in 6-well plates which were precoated with $0.01 \%$ poly-L-lysine and cultured in DMEM containing 10\% FBS and 1\% penicillin-streptomycin solution (Solarbio) in an atmosphere of $5 \% \mathrm{CO}_{2}$ and $95 \%$ air at $37^{\circ} \mathrm{C}$. All experiment procedures were carried following the ethical standards under a protocol approved by the Animal Experiment Ethics Committee of North China University of Science and Technology, and were executed conforming to the Guide for the Care and Use of Laboratory Animals published by the US National Institutes of Health (No. 85-23, 1996) [17].

The in vitro IR model was achieved by oxygen-glucose deprivation/reoxygenation (OGDR). Briefly, the primary hippocampal neurons were seeded in the 6-well plate at the density of $1 \times 10^{6}$ cells/well and the medium was changed to glucose-free DMEM in an atmosphere of $1 \% \mathrm{O}_{2}, 94 \% \mathrm{~N}_{2}$ and $5 \% \mathrm{CO}_{2}$ (hypoxic condition) at $37^{\circ} \mathrm{C}$ for $3 \mathrm{~h}$. Thereafter, the neurons were cultured in normal DMEM in an atmosphere of $95 \%$ air and $5 \% \mathrm{CO}_{2}$ (normoxic condition) up to $48 \mathrm{~h}$. Neurons cultured in normal medium under normoxic condition were used as control group.

Transfection was performed by mixing miR-378a-5p mimics, miR-378a-5p inhibitors and their negative controls (Ribobio, Guangzhou, China), and shCAMKK2 and its negative control (BersinBio, Guangzhou, China) with lipofectamine 2000 (Invitrogen, Carlsbad, WA, USA) according to manufacturer's protocols. The primary hippocampal neurons were cultured in the 6-well plate at the density of $1 \times 10^{6} \mathrm{cells} /$ well with FBS-free medium. The transfection complex was added into neurons and incubated for $6-8 \mathrm{~h}$. The medium was then replaced by normal culture medium. Neurons were cultured for $24 \mathrm{~h}$ at $37^{\circ} \mathrm{C}$ and collected for further analysis.

Determination of cell viability and cell survival. $M T T$ assay. The neurons were cultured in 96-well plates. After transfection, MTT $(5 \mathrm{mg} / \mathrm{ml}$ in phosphate-buffered saline (PBS, Abcam, Cambridge, UK) was added into each well and incubated for $4 \mathrm{~h}$ following the manufacturer's instruction. The formazan was dissolved by dimethyl sulfoxide. The absorbance value at $490 \mathrm{~nm}$ was measured and recorded by a spectrophotometer (BioTek, Winooski, VT, USA).

Trypan blue assay. Cell survival was determined using Trypan blue assay. The neurons were cultured in 6-well plate. After transfection, the medium was removed and cells were washed using PBS. $0.4 \%$ of trypan blue solution (SigmaAldrich, St. Louis, MO, USA) was added into each well at a ratio of 1:9 vs. PBS. The results were observed 
and photographs were acquired under a microscope within 3 min. All the cells visible in the view field were counted in tree view fields in each experimental group.

Apoptosis measurement by flow cytometry. The neurons were cultured in 6-well plates. After transfection, cells were suspended in $1.5 \mathrm{ml}$ of tubes and washed with PBS. The suspended cells were stained by the commercial FITC/PI apoptosis detection kit (Beyotime) according to the manufacturer's protocol. Cell apoptosis was analyzed with a flow cytometer (Becton Dickinson, Mountain View, CA, USA) by two parameter dot-plots. A total of 20,000 cells were recorded in each run.

Luciferase reporter assay. HEK293 cells were cultured in 6-well plates at the density of $1 \times 10^{6}$ cells/well. 3'-UTR of CAMKK2 with wild type sequence (CAMKK2-WT) and mutant sequence (CAMKK2-MUT) of miR-378a-5p complementary sites were cloned into pGL3 luciferase reporter vectors (Promega, Madison, WI, USA) respectively. pGL3CAMKK2-WT or pGL3-CAMKK2-MUT was co-transfected with miR-378a-5p mimics and its negative control. Luciferase activity was detected using Luciferase Reporter Assay Kit (BioVision, Milpitas, CA, USA).

Extraction of RNA and quantitative real time PCR (qRTPCR) assay. Total RNA extraction was performed using TRIzol reagent (Thermo Fisher). Reverse transcription was performed with $1 \mu \mathrm{g}$ of RNA using QuantiTect Reverse Transcription Kit (QIAGEN, Dusseldorf, Germany). RNA expression was examined using QuantiTect SYBR Green RT-PCR Kit (QIAGEN) with the StepOnePlus system (Applied Biosystems, USA). Relative RNA expression was quantified by $2^{-\Delta \Delta C t}$ method. The primer sequences (SigmaAldrich) used in this study were: $\beta$-actin forward, 5'-AGCCTCGCCTTTGCCGA-3' and reverse, 5'-CTGGTGCCTGGGGCG-3'; CAMKK2 forward, 5'-CGGTCGCAAGCTGTCTCTG-3' and reverse, 5'-GCGTCCGTTCATGTCCAGG-3'; U6 forward, 5'-AGTAAGCCCTTGCTGTCAGTG-3' and reverse, 5'-CCTGGGTCTGATAATGCTGGG-3'. Relative expression of miR-378a-5p was determined using miScript Primer Assays (QIAGEN) and normalized by U6 small RNA.

Western blotting. Cell lysates was extracted by RIPA cell lysis buffer (Beyotime). Total proteins $(5 \mu \mathrm{g})$ were loaded in SDS-PAGE and separated via electrophoresis. The separated proteins then transferred to PVDF membrane followed by blockade of membranes with $5 \%$ of milk. The blocked membranes were probed with the proper primary antibodies at $4^{\circ} \mathrm{C}$ overnight and then incubated with secondary antibodies for $2 \mathrm{~h}$ at room temperature. The signal of protein bands was detected using ECL Detection reagents (SigmaAldrich, USA). The primary antibodies (Cell Signaling, Danvers, MA, USA) used in this study were: Bax (CST\#2774S, 1:500 dilution), cleaved caspase-3 (CST\#9664, 1:1000 dilution), caspase-3 (CST\#9662S, 1:1000 dilution), CAMKK2 (ab96531, 1:500 dilution, Abcam), p-AMPK (CST\#2535, 1:2000 dilution), AMPK (CST\#2532, 1:2000 dilution), $\beta$-actin (CST\#4970, 1:5000 dilution).

Statistical analysis. GraphPad Prism 8.0 software (Grapgpad Inc., San Diego, CA, USA) was used to carry out all the data analysis. Student $t$-test was used to compare the difference between two groups. One-way ANOVA was used to compare between multiple groups. All data were expressed as mean \pm SD. $p<0.05$ indicated statistically significant difference.

\section{Results}

\section{miR-378a-5p was overexpressed after OGDR in primary hippocampal neurons}

After OGDR, overexpression of miR-378a-5p was detected at $24 \mathrm{~h}$ and $48 \mathrm{~h}$ compared with control group (Fig. 1). No change of miR-378a-5p was observed in the control group. The expression of miR-378a-5p was upregulated by OGDR in a time-dependent manner (Fig. 1).

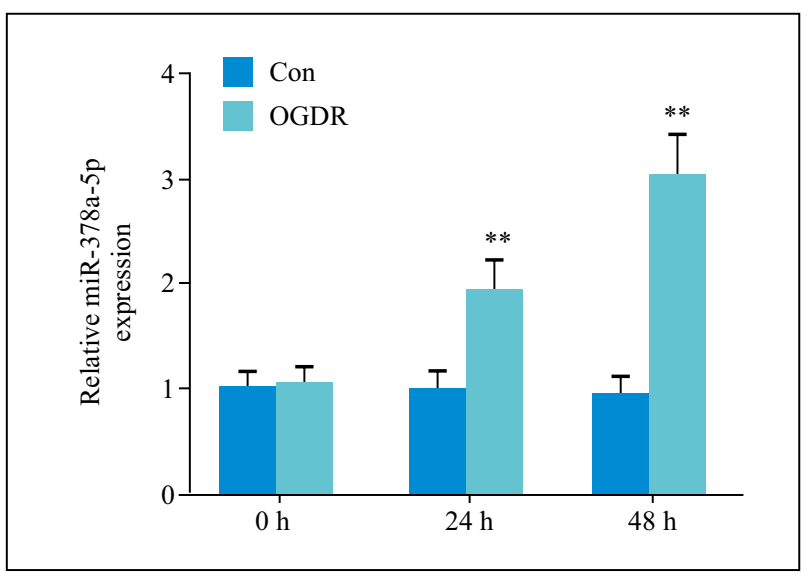

Figure 1. Expression of miR-378a-5p was upregulated in primary hippocampal neurons after OGDR. Primary hippocampal neurons were cultured in glucose-free DMEM in an atmosphere of $1 \% \mathrm{O}_{2}, 94 \% \mathrm{~N}_{2}$ and $5 \% \mathrm{CO}_{2}$ (hypoxic condition) at $37^{\circ} \mathrm{C}$ for $3 \mathrm{~h}$. Thereafter, the neurons were cultured in normal DMEM in an atmosphere of $95 \%$ air and $5 \% \mathrm{CO}_{2}$ (normoxic condition) for $48 \mathrm{~h}$. Neurons cultured in normal medium under normoxic condition were used as control group. Relative expression of miR-378a-5p after OGDR was detected by qRT-PCR as described in Methods. ${ }^{* *} p<0.01 v s$. control group. Abbrev.: Con - control; OGDR — oxygen-glucose deprivation/reoxygenation. 


\section{Inhibition of miR-378a-5p promoted cell proliferation and suppressed cell apoptosis induced by $O G D R$}

To investigate the role of miR-378a-5p in OGDR-induced neuron dysfunction, miR-378a-5p inhibitors were used to knockdown the expression of miR-378a$5 \mathrm{p}$. qRT-PCR results demonstrated that the expression of miR-378a-5p was lower in cells transfected with miR-378a-5p inhibitors than that in control group (Fig. 2A). After OGDR, cell viability was significantly reduced compared with the normoxic group and the reduction of cell viability was restored by miR-378a-5p inhibitors (Fig. 2B). mir-378a-5p inhibitor had no significant effects on the cell viability under normoxic condition (Fig. 2B). After OGDR, cell survival was significantly decreased compared with the normoxic group and this reduction of cell survival was attenuated by miR-378a-5p inhibitors (Fig. 2C). Mir-378a-5p inhibitor had no effects on the cell survival under normoxic condition (Fig. 2C). After OGDR, protein expressions of Bax and cleaved caspase-3 were upregulated compared with normoxic group and this upregulation of protein expression was attenuated by miR-378a-5p inhibitors (Fig. 2D). There was no significant change of Bax and cleaved caspase-3 expression between cell transfected with miR-378a-5p inhibitors and its negative controls under normoxic condition (Fig. 2D). After OGDR, cell apoptosis was significantly induced compared with the normoxic group and this induction of cell apoptosis was restored by miR-378a-5p inhibitors (Fig. 2E). Mir-378a-5p inhibitor did not show any effects on the cell apoptosis under normoxic condition (Fig. 2E).

\section{CAMKK2 is the direct target of $\mathrm{miR}-378 a-5 p$}

Prediction results from TargetScan (www.targetscan. org) demonstrated that there was a complementary sequence between 3'-UTR of CAMKK2 and miR378a-5p (Fig. 3A). Luciferase assay results showed that luciferase activity was significantly reduced in cells co-transfected with CAMKK2-WT plasmid and miR-378a-5p mimics while no change was observed in cells co-transfected with CAMKK2-MUT plasmid and miR-378a-5p mimics (Fig. 3B). The expression of miR-378a-5p was increased by miR-378a-5p mimics and decreased by miR-378a-5p inhibitors (Fig. 3C). The expression of CAMKK2 mRNA was downregulated by miR-378a-5p mimics and upregulated by miR-378a-5p inhibitors (Fig. 3D). Protein expression of CAMKK2 was also downregulated by miR-378a-5p mimics and upregulated by miR-378a-5p inhibitors (Fig. 3E). These results suggested that CAMKK2 is the direct target of miR-378a-5p.

\section{Effects of miR-378a-5p on primary hippocampal neurons were mediated by the inhibition of CAMKK2}

After OGDR for $48 \mathrm{H}$, the relative expression of CAMKK2 was reduced compared with control group. This reduction was prevented by miR-378a$5 p$ inhibitors and further reduced in cells transfected with shCAMKK2, which was reversed by miR-378a-5p inhibitors (Fig. 4A). Cell viability was also decreased after OGDR, and this decrease was suppressed by miR-378a-5p inhibitors and enhanced in cells transfected with shCAMKK2 (Fig. 4B). Inhibition of cell viability by shCAMKK2 was prevented in cells co-transfected with shCAMKK2 and miR-378a-5p inhibitors (Fig. 4B). Cell survival was also reduced after OGDR, and this reduction was suppressed by miR-378a-5p inhibitors and enhanced in cells transfected with shCAMKK2 (Fig. 4C). Inhibition of cell viability by shCAMKK2 was prevented in cells co-transfected with shCAMKK2 and miR-378a-5p inhibitors (Fig. 4C). The expressions of CAMKK2 and phosphorylation of AMPK were downregulated after OGDR, which were reversed by miR-378a-5p inhibitors (Fig. 4D). shCAMKK2 further reduced expression of CAMKK2 and phosphorylation of AMPK, which was inhibited by dual-inhibition of CAMKK2 and miR-378a-5p (Fig. 4D). Upregulation of Bax and cleaved caspase-3 was observed after OGDR and miR-378a-5p inhibitors repressed the upregulation of Bax and cleaved caspase- 3 induced by OGDR (Fig. 4D). Expression of Bax and cleaved caspase- 3 was further increased in cells transfected with shCAMKK2 and dual repression of CAMKK2 whereas miR-378a$5 \mathrm{p}$ prevented the overexpression of Bax and cleaved caspase- 3 induced by shCAMKK2 (Fig. 4D). Cell apoptosis was also induced after OGDR, and this induction was suppressed by miR-378a-5p inhibitors while enhanced by transfection with shCAMKK2 (Fig. $4 \mathrm{E})$. Inhibition of cell viability by shCAMKK2 was prevented in cells co-transfected with shCAMKK2 and miR-378a-5p inhibitors (Fig. 4E).

\section{Discussion}

Oxygen-glucose deprivation (OGD) is an in vitro ischemia-reperfusion model which is usually used to mimic ischemic cell damage [18]. Sustained OGD (lasting more than 1 hour) followed by re-oxygenation (OGDR) has been proved to induce mitochondria dysfunction, ROS production, and cell necrosis [18]. In this study, the expression of miR-378a-5p was significantly increased after OGDR in primary rat hippocampal neurons. Overexpression of miR-378a$5 p$ inhibited cell viability and promoted cell apoptosis 


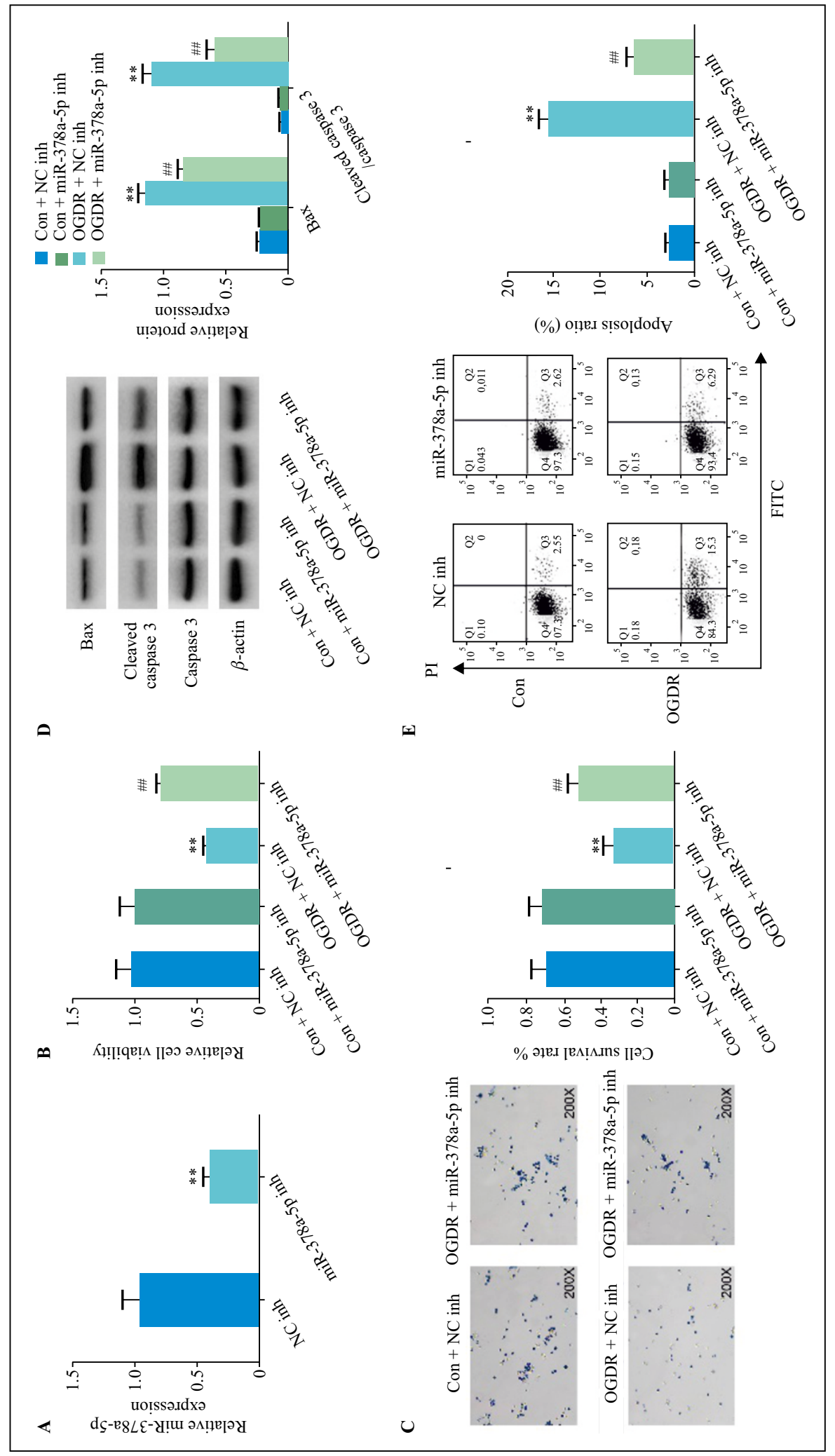

Figure 2. Inhibition of miR-378a-5p promoted neuronal cells' proliferation and suppressed cell apoptosis induced by OGDR. Incubation conditions were the same as described in the legend to Figure 1. A. miR-378a-5p expression was downregulated by miR-378a-5p inhibitors $48 \mathrm{~h}$ after OGDR. B. miR-378a-5p inhibitors prevented the reduction of cell viability after OGDR. C. miR-378a-5p inhibitors prevented the reduction of cell survival after OGDR. D. Western blotting revealed that miR378a-5p inhibitors inhibited the upregulation of Bax and cleaved caspase- 3 induced by OGDR. E. miR-378a-5p inhibitors prevented the induction of cell apoptosis after OGDR. ${ }^{* *} p<0.01 v s$. control cells $+\mathrm{NC}$ inh; ${ }^{\#} p<0.01 v s$. OGDR + NC inh. Abbrev.: as in the legend to Figure 1 plus NC inh, negative control of miR-378a-5p inhibitors. 


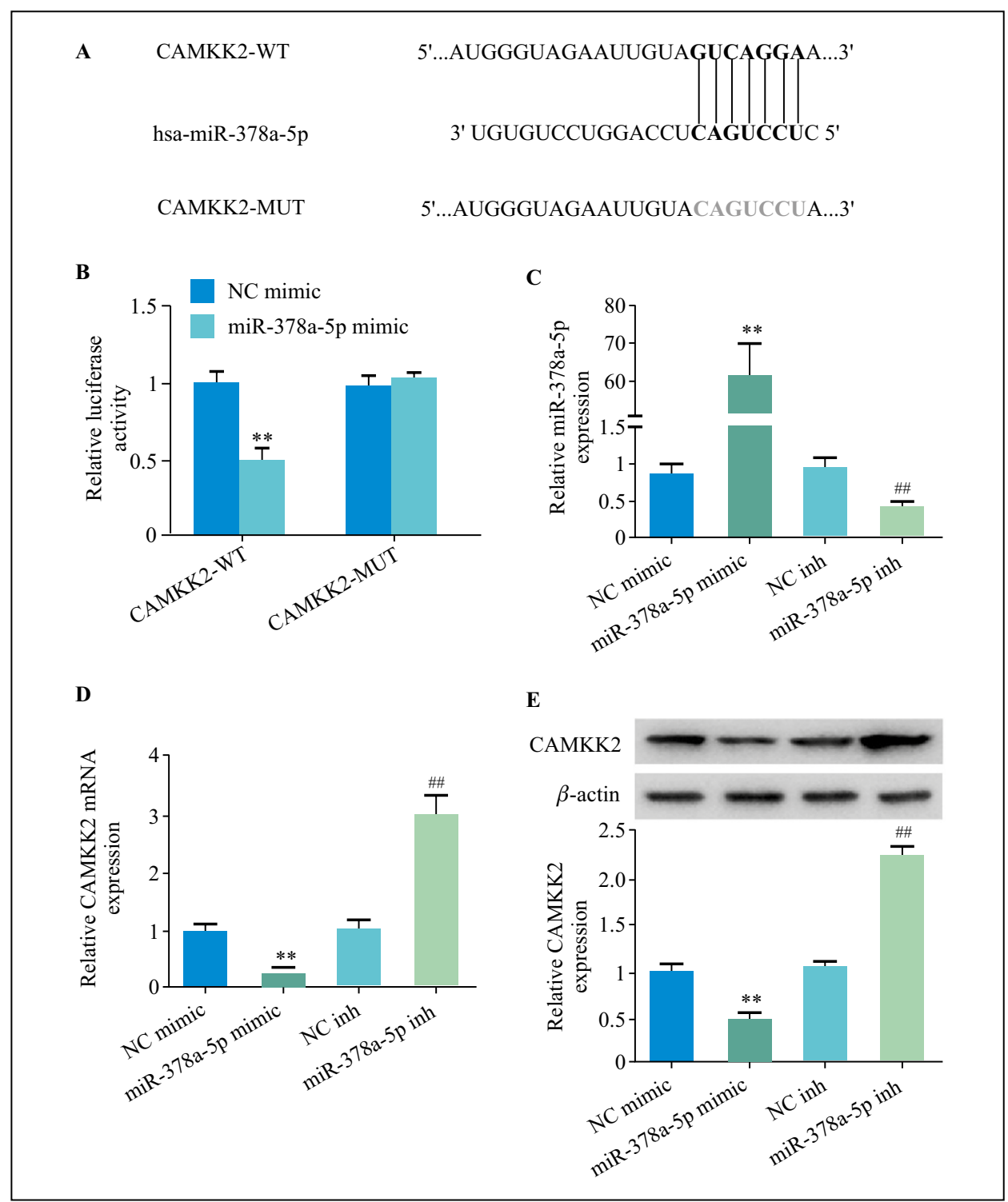

Figure 3. Calmodulin-dependent protein kinase kinase-2 (CAMKK2) is the direct target of miR-378a-5p in hippocampal neurons. Primary hippocampal neurons were incubated in the same conditions as described in the legend to Figure 1 . A. Predicting binding site of miR-378a 5p to 3'-UTR of CAMKK2. B. miR-378a-5p reduced the luciferase activity in CAMKK2 amplified cells. C. Expression of miR-378a-5p was increased by miR-378a-5p mimics and decreased by miR-378a-5p inhibitors $48 \mathrm{~h}$ after OGDR. D. Expression of CAMKK2 mRNA was downregulated by miR-378a-5p mimics and upregulated by miR-378a-5p inhibitors. E. Expression of CAMKK2 protein was downregulated by miR-378a-5p mimics and upregulated by miR-378a-5p inhibitors. **p $<0.01$ vs. NC mimic; ${ }^{\# \# p}<0.01$ vs. NC inh. Abbrev.: NC mimic - negative control of miR378a-5p mimic. WT: 3'-UTR of CAMKK2 with wild type sequence, MUT: 3'-UTR of CAMKK2 with mutant sequence.

through directly targeting 3'-UTR of CAMKK2 by activation of AMPK signaling pathway. Suppression of miR-378a-5p and overexpression of CAMKK2 could promote neuron viability and inhibit cell apoptosis, providing a new mechanism and biomarker for diagnosis and treatment of CIR injury.

Various miRNAs have been found to be involved in CIR injury. For example, elevation of miR-125-induced cell apoptosis and increased ROS production in PC-12 cells after OGDR were found to act via inhibition of casein kinase $2 \alpha(\mathrm{CK} 2 \alpha) / \mathrm{NADPH}$ oxidase signaling pathway [19]. miR-182-5p, which was downregulated after OGD in microglial cells, could prevent CIR injury by direct binding to Toll-like receptor 4 , demonstrating a neuroprotective effect [20]. In this study, the expression of miR-378a-5p was increased after OGDR, resulting in the suppression of cell proliferation and induction of cell apoptosis which may contribute to cerebral IR injury. Inhibition of miR-378a-5p prevented neuronal apoptosis 


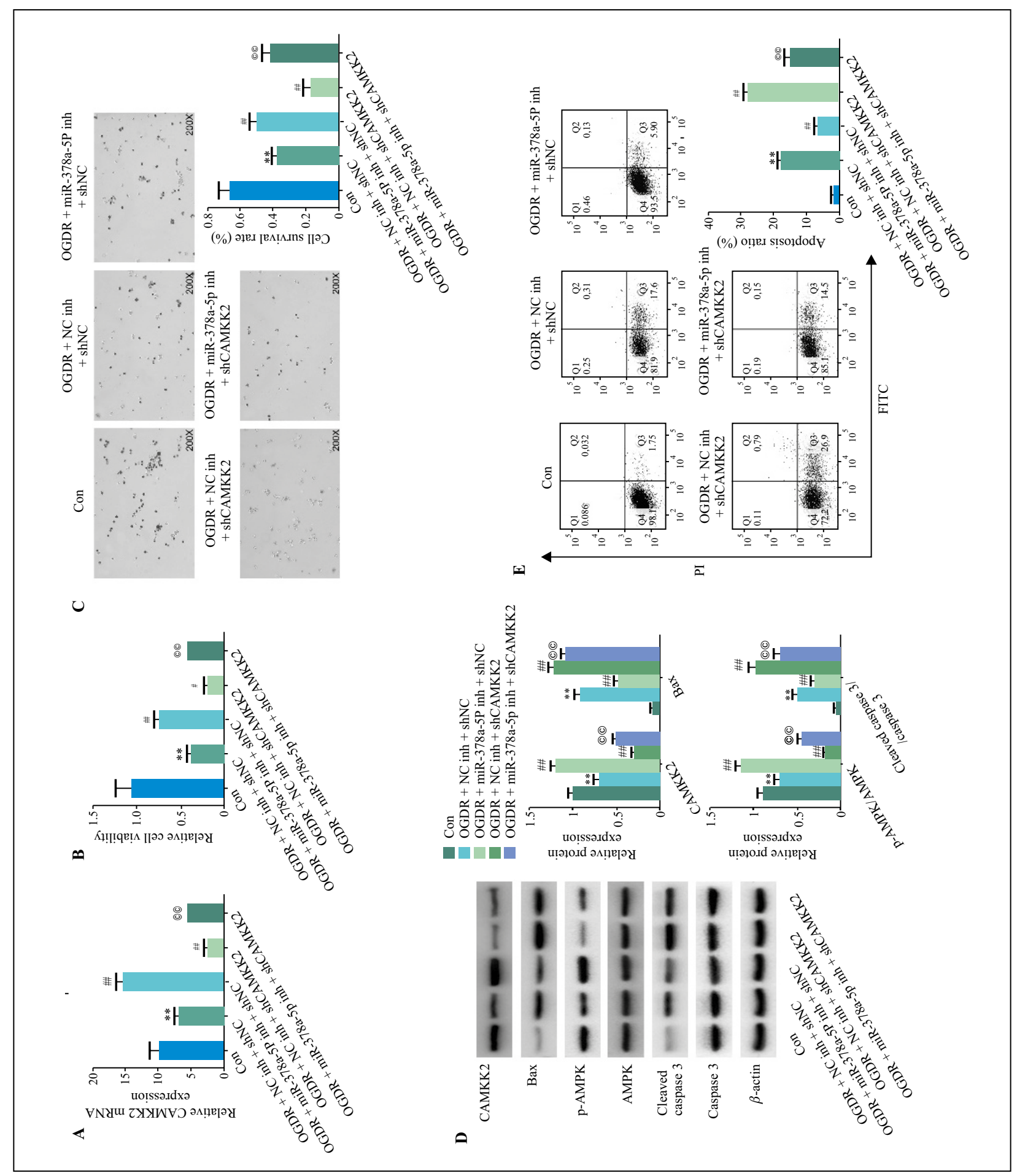

Figure 4. Effects of miR-378a-5p on primary hippocampal neurons was mediated by the inhibition of CAMKK2. The primary hippocampal neurons were cultured in glucose-free DMEM in an atmosphere of $1 \% \mathrm{O}_{2}, 94 \% \mathrm{~N}_{2}$ and $5 \% \mathrm{CO}_{2}$ (hypoxic condition) at $37^{\circ} \mathrm{C}$ for $3 \mathrm{~h}$. Thereafter, the neurons were cultured in normal DMEM in an atmosphere of $95 \%$ air and $5 \%$ $\mathrm{CO}_{2}$ (normoxic condition) for $48 \mathrm{~h}$. Neurons cultured in normal medium under normoxic condition were used as control group. A. Changes of CAMKK2 mRNA expression after treatment and transfection $48 \mathrm{~h}$ after OGDR. B. Change of cell viability after treatment and transfection $48 \mathrm{~h}$ after OGDR. C. Change of cell survival assessed by trypan blue staining after treatment and transfection. D. Change of CAMKK2, Bax, p-AMPK and cleaved caspase 3 protein expression after treatment and transfection. E. Cell apoptosis was measured by flow cytometry after treatment and transfection as described in Methods. ${ }^{* *} \mathrm{p}<0.01$ vs. con; ${ }^{\# \#} p<0.01 v s$. OGDR + NC inh + shNC; ${ }^{\circledR}{ }^{\circledR} p<0.01 v s$. OGDR + NC inh + shCAMKK2. Abbrev.: shCAMKK2 - shRNA of CAMKK2; shNC — negative control of shCAMKK2. 
induced by OGDR, which provides a new insight for the treatment of CIR injury.

miR-378a-5p is encoded by Ppargc1b gene and has been reported to act as a tumor suppressor because it could inhibit cell proliferation and induce cell apoptosis [21]. For example, by binding to the 3'-UTR of suppressor of fused homolog (SUFU), miR-378a-5p promoted cell apoptosis in triple negative breast cancer [22]. In oral squamous cell carcinoma, miR-378a$5 \mathrm{p}$ was shown to be negatively associated with the cell proliferation and inhibited angiogenesis through binding to kallikrein-related peptidase 4 (KLK4), inhibiting tumorigenesis and tumor metastasis [23]. Therefore, the overexpression of miR-378a-5p improves the prognosis of cancer patients. However, the inhibitory effects of miR-378a-5p on cell survival play a negative role in cerebral IR injury. In this study, overexpression of miR-378a-5p reduced cell survival after OGDR, which worsens the prognosis of cerebral IR injury. Therefore, downregulation of miR-378a-5p could prevent cerebral IR injury.

As mentioned before, miRNA could bind to 3'-UTR of target mRNA to induce translational repression. There are target regions called "seed regions", which refer to the 7 nucleotides at 2 to 8 at the 5 " end of miRNA [12]. The "seed region" is the key sequence to recognize the target of miRNAs [12]. The prediction results in this study from TargetScan indicated that there was a complementary sequence between 3'-UTR of CAMKK2 and 2 to 8 at the 5" end of miR-378a-5p in compliance with Watson-Crick match [24], suggesting that calmodulin-dependent protein kinase kinase-2 was the direct target of miR-378a$5 \mathrm{p}$. Luciferase activity was significantly reduced in cells co-transfected with CAMKK2-WT plasmid and miR-378a-5p mimics while no change was observed in cells co-transfected with CAMKK2-MUT plasmid and miR-378a-5p mimics, further confirming that CAMKK2 was a direct target of miR-378a-5p.

CAMKK2 is a $\mathrm{Ca}^{2+} /$ calmodulin-dependent serine-threonine protein kinase which could couple calcium transients to regulate cell proliferation, survival and metabolism [25]. Activation of CAMKK2 by phosphorylation of AMPK on Thr172, results in inhibition of protein and lipid synthesis, and suppression of cell growth $[8,9]$. Data of this study show that inhibition of CAMKK2 prevented cell growth induced by the downregulation of miR-378a-5p, indicating that inhibition of miR-378a-5p could prevent neuronal apoptosis during CIR injury. Furthermore, inhibition of CAMKK2 reduced the phosphorylation of AMPK. All these effects were attenuated by dual-inhibition of miR-378a-5p and CAMKK2, suggesting that miR378a is involved in CIR through regulation of CAM-
KK2-AMPK signaling pathway and that CAMKK2 showed an neuroprotective effect during CIR injury. In conclusion, data of this study show that miR-378a$5 p$ was significantly overexpressed after OGDR in primary rat hippocampal neurons, providing a new biomarker for cerebral IR injury. miR-378a-5p could inhibit cell proliferation and induce cell apoptosis through regulation of CAMKK2/AMPK pathway, providing a new therapeutic target for CIR injury. However, the findings of this study should be confirmed in clinical practice and future drug discovery could focus on the activation of CAMKK2/AMPK pathway.

\section{Acknowledgements}

Not applicable.

\section{Funding}

Not applicable.

\section{Competing interests}

The authors state that there are no conflicts of interest to disclose.

\section{Ethics approval}

See material and methods section.

\section{Statement of Informed Consent}

Not applicable.

\section{Authors' contributions}

Yun Zhang designed the study, supervised the data collection, analyzed the data, Peilan Zhang interpreted the data and prepare the manuscript for publication, Chunying Deng supervised the data collection, analyzed the data and reviewed the draft of the manuscript. All authors have read and approved the manuscript.

\section{Availability of data and materials}

All data generated or analyzed during this study are included in this published article.

\section{References}

1. Sarkar S, Chakraborty D, Bhowmik A, et al. Cerebral ischemic stroke: cellular fate and therapeutic opportunities. Front Biosci (Landmark Ed). 2019; 24: 435-450, indexed in Pubmed: 30468665. 
2. Turley KR, Toledo-Pereyra LH, Kothari RU. Molecular mechanisms in the pathogenesis and treatment of acute ischemic stroke. J Invest Surg. 2005; 18(4): 207-218, doi: 10.1080/08941930591004449, indexed in Pubmed: 16126632.

3. Yang J, Chen M, Cao RY, et al. The Role of Circular RNAs in Cerebral Ischemic Diseases: Ischemic Stroke and Cerebral Ischemia/Reperfusion Injury. Adv Exp Med Biol. 2018; 1087: 309-325, doi: 10.1007/978-981-13-1426-1_25, indexed in Pubmed: 30259377.

4. Wu MY, Yiang GT, Liao WT, et al. Current mechanistic concepts in ischemia and reperfusion injury. Cell Physiol Biochem. 2018; 46(4): 1650-1667, doi: 10.1159/000489241, indexed in Pubmed: 29694958.

5. Wang JF, Mei ZG, Fu Y, et al. Puerarin protects rat brain against ischemia/reperfusion injury by suppressing autophagy the AMPK-mTOR-ULK1 signaling pathway. Neural Regen Res. 2018; 13(6): 989-998, doi: 10.4103/1673-5374.233441, indexed in Pubmed: 29926825.

6. Curry DW, Stutz B, Andrews ZB, et al. Targeting AMPK Signaling as a neuroprotective strategy in Parkinson's disease. J Parkinsons Dis. 2018; 8(2): 161-181, doi: 10.3233/JPD171296, indexed in Pubmed: 29614701.

7. Woods A, Johnstone SR, Dickerson K, et al. LKB1 is the upstream kinase in the AMP-activated protein kinase cascade. Curr Biol. 2003; 13(22): 2004-2008, doi: 10.1016/j. cub.2003.10.031, indexed in Pubmed: 14614828.

8. Hawley SA, Pan DA, Mustard KJ, et al. Calmodulin-dependent protein kinase kinase-beta is an alternative upstream kinase for AMP-activated protein kinase. Cell Metab. 2005; 2(1): 9-19, doi: 10.1016/j.cmet.2005.05.009, indexed in Pubmed: 16054095.

9. Penfold L, Woods A, Muckett P, et al. CAMKK2 promotes prostate cancer independently of AMPK via increased lipogenesis. Cancer Res. 2018; 78(24): 6747-6761, doi: 10.1158/0008-5472.CAN-18-0585, indexed in Pubmed: 30242113.

10. Ma C, Wang X, Xu T, et al. Qingkailing injection ameliorates cerebral ischemia-reperfusion injury and modulates the AMPK/NLRP3 inflammasome signalling pathway. BMC Complement Altern Med. 2019; 19(1): 320, doi: 10.1186/ s12906-019-2703-5, indexed in Pubmed: 31747940.

11. Du S, Deng Y, Yuan H, et al. Safflower yellow B protects brain against cerebral ischemia reperfusion injury through AMPK/NF-kB pathway. Evid Based Complement Alternat Med, 2019; 2019: 1-11, doi: 10.1155/2019/7219740, indexed in Pubmed: 30854014.

12. Liu Xi, Chen L, Liu Y, et al. Tangeretin sensitises human lung cancer cells to TRAIL induced apoptosis via ROS-JNK/ ERK-CHOP pathway mediated up-regulation of death receptor 5. Tropical J Pharmac Res. 2017; 16(1): 17, doi: 10.4314/ tjpr.v16i1.4.

13. Di Yu, Lei Y, Yu F, et al. MicroRNAs expression and function in cerebral ischemia reperfusion injury. J Mol Neurosci.
2014; 53(2): 242-250, doi: 10.1007/s12031-014-0293-8, indexed in Pubmed: 24696166.

14. Liu S, Yang Y, Jiang S, et al. Corrigendum: MiR-378a-5p regulates proliferation and migration in vascular smooth muscle cell by targeting CDK1. Front Genet. 2019; 10: 193, doi: 10.3389/fgene.2019.00193, indexed in Pubmed: 30930936 ,

15. Gao W, Zhou X, Lin R. miR-378a-5p and miR-630 induce lens epithelial cell apoptosis in cataract via suppression of E2F3. Braz J Med Biol Res. 2020; 53(5): e9608, doi: 10.1590/1414431x20209608, indexed in Pubmed: 32348429.

16. Li L, Liu Z, Jiang YY, et al. Acetylcholine suppresses microglial inflammatory response via $\alpha 7 \mathrm{nAChR}$ to protect hippocampal neurons. J Integr Neurosci. 2019; 18(1): 51-56, doi: 10.31083/j.jin.2019.01.114, indexed in Pubmed: 31091848 .

17. Council NR. Guide for the care and use of laboratory animals: eighth edition. 2010. 327(3): 963-965.

18. Zheng K, Zhang Q, Lin G, et al. Activation of Akt by SC79 protects myocardiocytes from oxygen and glucose deprivation (OGD)/re-oxygenation. Oncotarget. 2017; 8(9): 1497814987, doi: 10.18632/oncotarget.14785, indexed in Pubmed: 28122357.

19. Liang Y, Xu J, Wang Yu, et al. Inhibition of MiRNA-125b decreases cerebral ischemia/reperfusion injury by targeting CK $2 \alpha /$ NADPH oxidase signaling. Cell Physiol Biochem. 2018; 45(5): 1818-1826, doi: 10.1159/000487873, indexed in Pubmed: 29510389.

20. Wang Ji, Xu Z, Chen X, et al. MicroRNA-182-5p attenuates cerebral ischemia-reperfusion injury by targeting Toll-like receptor 4. Biochem Biophys Res Commun. 2018; 505(3): 677-684, doi: 10.1016/j.bbrc.2018.09.165, indexed in Pubmed: 30292407.

21. Machado IF, Teodoro JS, Palmeira CM, et al. miR-378a: a new emerging microRNA in metabolism. Cell Mol Life Sci. 2020; 77(10): 1947-1958, doi: 10.1007/s00018-019-03375-z, indexed in Pubmed: 31748917.

22. Zheng S, Li M, Miao K, et al. IncRNA GAS5-promoted apoptosis in triple-negative breast cancer by targeting miR-378a5p/SUFU signaling. J Cell Biochem. 2020; 121(3): 2225-2235, doi: 10.1002/jcb.29445, indexed in Pubmed: 31692053.

23. Cui Z, Liu QL, Sun SQ, et al. MiR-378a-5p inhibits angiogenesis of oral squamous cell carcinoma by targeting KLK4. Neoplasma. 2020; 67(1): 85-92, doi: 10.4149/neo_2019_190306N191, indexed in Pubmed: 31829025.

24. Varani G, McClain WH. The Gx U wobble base pair. A fundamental building block of RNA structure crucial to RNA function in diverse biological systems. EMBO Rep. 2000; 1(1): 18-23, doi: 10.1093/embo-reports/kvd001, indexed in Pubmed: 11256617.

25. Racioppi L, Nelson ER, Huang W, et al. CaMKK2 in myeloid cells is a key regulator of the immune-suppressive microenvironment in breast cancer. Nat Commun. 2019; 10(1): 2450, doi: 10.1038/s41467-019-10424-5, indexed in Pubmed: 31164648 . 